\title{
Mapping Out Receptors: High Throughput Screening of Endothelial Monocyte Activating Polypeptide II (EMAPII) Using PRESTO-TANGO
}

\author{
Nathan Lam ${ }^{1}$, Bernhard Maier ${ }^{2}$, Sarvesh Chelvanambi ${ }^{3}$, Takashi Hato ${ }^{2}$ and \\ Matthias Clauss ${ }^{3}$ \\ ${ }^{1}$ Indiana University School of Medicine ${ }^{2}$ Indiana University School of Medicine, \\ Department of Medicine, Division of Pulmonary and Critical Care \\ Medicine ${ }^{3}$ Indiana University School of Medicine, Department of Medicine, \\ Division of Pulmonary and Critical Care
}

Background: Secreted endothelial monocyte activating polypeptide II (EMAPII/AIMP1) is a pro-apoptotic, pro-inflammatory ligand implicated in diseases such as colorectal cancer, cardiovascular disease, and emphysema. Thus, EMAPII has been shown to induce apoptosis through CXCR3 receptor binding. However, not all EMAPII functions have been attributed to CXCR3. Discovery of new receptors interacting with EMAPII could lead to development of new therapies blocking cognate ligand-receptor binding. We hypothesize the existence of at least one unknown secondary receptor for EMAPII.

Methods: The PRESTO-TANGO assay, a construct which converts G-protein coupled receptor (GPCR) ligand binding into luciferase activity measurable by luminometer, was validated using transfection with TANGO-modified CXCR3 and S1P1R as test receptors in HTLA cells. Protocols for cell transfection, adherence, and cultivation were optimized with IP10, EMAPII, and S1P as test ligands.

Results: The assay was successfully validated using several GPCR activation readouts. Binding curves were generated for S1P/S1P1 $\left(E_{50}=569 \mathrm{nM}\right)$, IP10/CXCR3 $\left(E_{50}=47.1 \mathrm{nM}\right)$, and EMAPII/CXCR3 $\left(\mathrm{EC}_{50}=628 \mathrm{nM}\right)$. Conditions for the PRESTO-TANGO assay were further refined for maximal signal-to-noise ratio and robust inter-assay reproducibility in preparation for high-throughput screening. We are currently testing 314 TANGO-modified GPCRs for EMAPII affinity.

Conclusion: We have validated the Tango assay for the known EMAPII-CXCR3 ligand-receptor system, a valuable tool for evaluation of anti-EMAPII therapeutics. Discovery of a novel EMAPII receptor would allow for the development of therapies including neutralizing antibodies (analogous to the PD1 receptor antibody Pembrolizumab for solid tumors) in diseases such as colorectal cancer, cardiovascular disease, and emphysema. 